\title{
Integrated Electronics in the Car and the Design Chain Evolution or Revolution?
}

\author{
Alberto Sangiovanni-Vincentelli \\ The Edgar L. and Harold H. Chair of EECS, University of California at Berkeley \\ Chief Technology Advisor, Cadence Design Systems
}

\section{Setting the Stage}

The lack of an overall understanding of the interplay of the sub-systems and of the difficulties encountered in integrating very complex parts, system integration is becoming increasingly a nightmare. In fact, Jurgen Hubbert, in charge of the Mercedes-Benz passenger car division, publicly stated in 2003: "The industry is fighting to solve problems that are coming from electronics and companies that introduce new technologies face additional risks. We have experienced blackouts on our cockpit management and navigation command system and there have been problems with telephone connections and seat heating." I believe that this sorry state is the rule for the leading OEMs, it is not the exception in todays environment. The source of these problems is clearly the increased complexity but also the difficulty of the OEMs in managing the integration and maintenance process with subsystems that come from different suppliers who use different design methods, different software architecture, different hardware platforms, different (and often proprietary) Real-Time Operating Systems. Therefore, the need for for standards in the software and hardware domains that will allow plug-and-play of subsystems and their implementation are essential while the competitive advantage of an OEM will increasingly reside on essential functionalities (e.g. stability control).

To deliver more performing, less expensive, and safer cars with increasingly tighter time-to-market constraints imposed by worldwide competitiveness, the future development process for automotive electronic systems must provide solutions to:

- The design of complex functionality with tight requirements on safety and correctness;

- The design of distributed architectures consisting of several subsystems with constraints on non functional metrics such as cost, power consumption, weight, position, and reliability;

- The mapping of the functionality (often implemented as OEM application Software) onto the components of a distributed architecture with tight real-time and communication constraints.

\section{The Challenges}

The Design and Supply chains are the backbone for any industrial sector. Their health and efficiency are essential for economic viability and profit maximization. While tools for Supply Chain management have been around for quite some time, support for the Design Chain has not been pursued nearly as vigorously. There are strong opportunities for improving the situation substantially at least in the automotive electronics industrial sector with a combination of tools and methodologies. However, we are just at the beginning. Integration of electronic and mechanical design tools and frameworks will be essential in the near future. Data integration and information flow among the companies forming the chain have to be supported. In other words, it is essential that the fundamental steps, that is, functional partitioning, allocation on computational resources, integration, and verification be supported across the entire V-Cycle. Thus, whether the integrations pertains to SW-SW integration on a distributed network, HW-SW integration on an ECU, electronics and mechanical integration for a sub-system, tools and models have to be integrated seamlessly from a static point of view (data dictionaries, off-line model transformations) and dynamic point of view (e.g. co-simulation and HW-in-the-loop simulations).

Assuming the design methodology and the infrastructure for design chain integration are all in place, what will be the implication on the industrial segment structure? Today, the roles of car makers, Tier 1 and 2 Suppliers are relatively stable but they are undergoing a period of stress due to the increased importance of electronics and its added value. We mention the desire of car makers to gain a stronger grip on the integration process and on the critical parts of the electronics subsystems. At the same time, there is evidence that sharing IPs among car makers and Tier 1 suppliers could improve substantially time-to-market, development and maintenance costs. The essential technical problem to 
solve for this vision is the establishment of standards for interoperability among IPs and tools. AUTOSAR has this goal very clear in mind. However, there are technical and business challenges to overcome. In particular, from the technical point of view, while sharing algorithms and functional designs seems feasible at this time once the semantic platform issues are squared away, the sharing of real-time software is difficult even assuming substantial improvements in design methods and technology, if run-time efficiency has to be retained. The issues are related to the interplay that different tasks can have at the RTOS level. The timing of the software tasks depend on the presence or absence of other tasks. A scheduling policy that could prevent timing variability in presence of dynamical changing task characteristics can be conceived but it will carry heavy overhead thus requiring powerful microprocessors even when they are not strictly needed. This is the standard trade-off between efficiency and reliability but it has more important business implications than usual. In fact, if software from different sources has to be integrated on a common hardware platform who will be responsible for the correct functioning of the final product?

Whoever will take on this responsibility would need a very strong methodology and an iron fist to make suppliers and partners comply with it. This may not be enough, in the sense that software characteristics are hard to pin down and with the best intentions of this world, one may not be able to guarantee functional AND timing behavior in the presence of foreign components. The ideal approach would be a tool that could map automatically the set of tasks onto the platform guaranteeing the correct functionality and timing with optimal resource utilization. This tool should take the design description at the pure functional level with performance and other constraints and the architecture of the platform and produce correct settings for the RTOS and optimized code. We are still far from this ideal. It is likely, then, that the responsibility for subsystem integration will still rest with the car manufacturers but the responsibility for integrating software components onto ECUs will be assigned to Tier 1 suppliers. In this case, the burden of Tier 1 suppliers will be increased at a possibly reduced premium because of the perceived reduction in added value. This is likely to be an unstable model and major attention should be devoted to find a common ground where both car makers and suppliers find their economic return.

If the strategy followed by car makers in AUTOSAR succeeds, then it is likely that a global restructuring of the industry will take place by creating an environment where Tier 1 players with small market share will find themselves in a difficult position unless they find a way of competing on a more leveled ground with the major stake holders. In this scenario, Tier 2 suppliers including IP providers may find themselves in a better position to entertain business re- lations directly with the car manufacturer. Tool providers will be in a more strategic position as providers of mapping tools that make the business model feasible. Hence, it is likely that a shift of recognized value will take place from Tier 1 suppliers towards tool providers and Tier 2 suppliers. The redistribution of wealth in the design chain may or may not be a positive outcome for the health of the industrial sector. If the discontinuities are sharp, then there may be a period of instability where much effort will be required to keep the products coming out with quality and reliability problems that may be larger than the ones observed lately. However, if it is well managed, then a natural shake-up with stronger players emerging will have a double positive: more quality in the products at lower cost. An additional benefit from a real plug-and-play environment will be the acceleration of the rate of innovation. Today, the automotive sector is considered conservative and the innovations in design methods and electronic components are slow to come. For example, if a well-oiled mechanism existed to migrate from one hardware platform to another, the optimal solutions would be selected instead of the ones that have been traditionally used. In this case, the Tier 2 market place will also be rationalized and the rate of innovation will likely be increased.

As a final consequence, the introduction of new functionalities will be a matter of algorithm and architecture rather than detailed software and hardware selection. The trend in electronics is clear: less customization, more standardization. This is indeed the reason why platform-based design and supporting tools [1] has appealed to a wide variety of electronic industry players. For a subsystem supplier, the choice will be richer in terms of platforms but it will not require heavy investment in IC design or RTOS development. For a car manufacturer, the granularity of its choices will be also richer because of interoperability. He will have the choice of selecting entire macro systems or components that could be integrated in a large automotive platform. The choice will be guided by cost, quality and product innovation.

The final goal of the strategy is rather clear. The way of getting there is not as clear and the road has many bumps and turns that are difficult to negotiate. A positive outcome will have to come from a process of deep business and technical cooperation among all players in the design chain as well as the research community. It is a unique opportunity and a great challenge.

\section{References}

[1] A. L. Sangiovanni-Vincentelli, L. Carloni, F. D. Bernardinis, and M. Sgroi. Benefits and challenges for platform-based design. Proceedings of the Design Automation Conference, June 2004. 